\title{
Differential equations for ruin probability in a special risk model with FGM copula for the claim size and the inter-claim time
}

Wu Yong ${ }^{1}$ and Hu Xiang ${ }^{2 *}$

\section{*Correspondence:}

huxiangaq@126.com

${ }^{2}$ Department of Risk Management

and Insurance, School of

Tianjin 300071, P.R. China

Full list of author information is

available at the end of the article
Economics, Nankai University,

\begin{abstract}
In this paper, we consider a risk process in which the distribution of the inter-claim time is the sum of two independent exponential random variables. We introduce a dependence structure between the claim size and the inter-claim time. The structure is based on FGM copula. An integro-differential equation for the expected discounted penalty function is derived and an explicit expression for the Laplace transform of ruin probability is given for exponential claim size.
\end{abstract}

\section{Introduction}

In classical risk models, the surplus process usually relies on the assumption of independence between the claim size and the inter-claim time. However, in many applications this assumption is too restrictive and unrealistic. Actually, we know that the greater the claim size is, the longer the inter-claim time is. The requirement for generalization has led to some papers on the modeling of dependence. Among them, Albrecher and Teugels [1] consider general dependence structure based on a copula for the claim size and the inter-claim time, they derive asymptotic results for both the finite and infinite time ruin probabilities. Boudreault et al. [2] consider a particular dependence structure among the inter-claim time and the claim size and derive the defective renewal equation satisfied by expected discounted penalty function. Cossette et al. [3] consider the compound Poisson risk model in which the claim size and the inter-claim time are dependent, and the dependence structure is based on Farlie-Gumbel-Morgenstern (FGM) copula. They derive the Laplace transform of the expected discounted penalty function, and give explicit expression for the Laplace transform of the time of ruin for exponential claim sizes. Barges and Cossette [4] investigate the computation of the moments of the compound Poisson risk model with FGM copula.

In this article, we consider a Sparre Andersen risk process where the claim size and the inter-claim time are dependent with FGM copula, and the distribution of the inter-claim time is the sum of two independent exponential random variables. In ruin theory, a common approach is to obtain an integro-differential equation for the expected discounted penalty function and apply it to derive the Laplace transform of the function. Dickson and Hipp [5] investigate ruin probability for Erlang(2) risk process, Li and Garrido [6] consider this problem of Erlang $(n)$ risk model, Gerber and Shui [7] also do some relative works.

(C) 2012 Yong and Xiang; licensee Springer. This is an Open Access article distributed under the terms of the Creative Commons Attribution License (http://creativecommons.org/licenses/by/2.0), which permits unrestricted use, distribution, and reproduction in any medium, provided the original work is properly cited. 
The paper is arranged as follows. In Section 2, we present the risk model and give some notations. An integro-differential equation of the expected discounted penalty function is formulated and the main results are presented in Section 3. In Section 4, we apply the integro-differential equation to derive the Laplace transform of the expected discounted penalty function. The special case where the claim size is exponentially distributed is considered in the final section.

\section{The risk model}

The surplus process is defined as $U(t)=u+c t-\sum_{i=1}^{N(t)} X_{i}$, where $U(0)=u$ is the initial surplus, $c$ is the premium rate, $\left\{N(t), t \in \mathbb{R}^{+}\right\}$is a renewal process, and $X_{i}(i=1,2, \ldots)$ is the random variable (r.v.) corresponding to the amount of the $i$ th claim. The time between the $(i-1)$ th and $i$ th claim is defined by the r.v. $V_{i}$ with $V_{1}$ being the time of the first claim.

The claim amounts $\left\{X_{i}, i \in \mathbb{N}^{+}\right\}$form a sequence of independent identically distributed (i.i.d.) random variables (r.v.s) as the r.v. $X$ with probability density function (p.d.f.) $f_{X}$, cumulative distribution function (c.d.f.) $F_{X}$. The inter-claim times $\left\{V_{i}, i \in \mathbb{N}^{+}\right\}$form a sequence of independent r.v.s identically distributed as the canonical r.v. $V, V=W_{1}+W_{2}$, where $\left\{W_{j}\right\}$ are two independent exponentially distributed r.v.s with parameters $\lambda_{j}, j=1,2$, $V$ has p.d.f. $f_{V}$, and c.d.f. $F_{V}$. Note that the $\operatorname{Erlang}(2)$ model is the special case where $\lambda_{1}=\lambda_{2}$. $\left\{\left(X_{i}, V_{i}\right), i \in \mathbb{N}^{+}\right\}$form a sequence of i.i.d. random vectors distributed as the canonical random vector $(X, V)$. The joint p.d.f. of $(X, V)$ is denoted by $f_{X, V}(x, t)$ with $x, t \in \mathbb{R}^{+}$.

The joint distribution of $(X, V)$ is based on the classical FGM copula, which is defined by

$$
C(u, v)=u v+\theta u(1-u) v(1-v)
$$

for every $(u, v)$ in $[0,1]^{2}$ and the dependence parameter $\theta$ takes value in $[-1,1]$.

We choose this class of copula since it provides an easy manner to construct bivariate models with a variety of dependence structures. Even if the FGM copula introduces only light dependence, it admits positive as well as negative dependence between a set of random variables and includes the independence copula when $\theta=0$ (see Nelsen [8]).

The p.d.f. associated to (1) is given by

$$
c(u, v)=1+\theta(1-2 u)(1-2 v) .
$$

Given (1), the joint c.d.f. $F_{X, V}$ is defined by

$$
\begin{aligned}
F_{X, V}(x, t) & =C\left(F_{X}(x), F_{V}(t)\right) \\
& =F_{X}(x) F_{V}(t)+\theta F_{X}(x)\left(1-F_{X}(x)\right) F_{V}(t)\left(1-F_{V}(t)\right) .
\end{aligned}
$$

With (2), the joint p.d.f. $f_{X, V}$ is

$$
\begin{aligned}
f_{X, V}(x, t) & =f_{X}(x) f_{V}(t) c\left(F_{X}(x), F_{V}(t)\right) \\
& =f_{X}(x) f_{V}(t)+\theta f_{X}(x) f_{V}(t)\left(1-2 F_{X}(x)\right)\left(1-2 F_{V}(t)\right) .
\end{aligned}
$$

For simplicity, we define the following functions:

$$
k_{X}(x)=f_{X}(x)\left(1-2 F_{X}(x)\right)
$$


and

$$
k_{V}(t)=f_{V}(t)\left(1-2 F_{V}(t)\right) .
$$

Let $T$ denote the time to ruin, so that

$$
T=\inf \{t \mid U(t)<0 \text {, for } t>0\} .
$$

Then the probability of ultimate ruin from initial surplus $u$ is defined as

$$
\psi(u)=P(T<\infty \mid U(0)=u) .
$$

To ensure that ruin will not occur almost surely, we assume that

$$
E(c V-X)>0
$$

Besides the ruin probability, other important ruin quantities in ruin theory include the Laplace transform of ruin time; the surplus immediately before ruin denoted by $U(T-)$; the deficit at ruin denoted by $|U(T)|$, etc. A unified approach to study these ruin quantities is to consider the so-called expected discounted penalty function introduced by Gerber and Shiu [9]; the function is given by

$$
m_{\delta}(u)=E\left[e^{-\delta T} \omega(U(T-),|U(T)|) I(T<\infty) \mid U(0)=u\right], \quad u>0,
$$

where $\omega(x, y)$, for all $x, y \geq 0$, is the penalty function at the time of ruin for the surplus prior to ruin and the deficit at ruin, $I(\cdot)$ is the indicator function, and $\delta$ is a nonnegative parameter. We can think of $\delta$ either as being a force of interest or as a dummy variable in the context of the Laplace transform. A special case of the expected discounted penalty function with $\omega(x, y)=1$ is the Laplace transform of the time of ruin. The cases $\delta=0$ and $\omega(x, y)=1$ correspond to the infinite-time ruin probability.

\section{Integro-differential equation}

In this section, we derive an integro-differential equation for the expected discounted penalty function $m_{\delta}(u)$. By conditioning on the time and the amount of the first claim, we have

$$
\begin{aligned}
m_{\delta}(u)= & \int_{0}^{\infty} \int_{0}^{u+c t} e^{-\delta t} m_{\delta}(u+c t-x) f_{X, V}(x, t) d x d t \\
& +\int_{0}^{\infty} \int_{u+c t}^{\infty} e^{-\delta t} \omega(u+c t, x-u-c t) f_{X, V}(x, t) d x d t
\end{aligned}
$$


With (3), (4) and (5), (8) becomes

$$
\begin{aligned}
m_{\delta}(u)= & \int_{0}^{\infty} \int_{0}^{u+c t} e^{-\delta t} m_{\delta}(u+c t-x) f_{X}(x) f_{V}(t) d x d t \\
& +\theta \int_{0}^{\infty} \int_{0}^{u+c t} e^{-\delta t} m_{\delta}(u+c t-x) k_{X}(x) k_{V}(t) d x d t \\
& +\int_{0}^{\infty} \int_{u+c t}^{\infty} e^{-\delta t} \omega(u+c t, x-u-c t) f_{X}(x) f_{V}(t) d x d t \\
& +\theta \int_{0}^{\infty} \int_{u+c t}^{\infty} e^{-\delta t} \omega(u+c t, x-u-c t) k_{X}(x) k_{V}(t) d x d t
\end{aligned}
$$

We define two functions:

$$
\sigma_{1}(y)=\int_{0}^{y} m_{\delta}(y-x) f_{X}(x) d x+\omega_{1}(y)
$$

and

$$
\sigma_{2}(y)=\int_{0}^{y} m_{\delta}(y-x) k_{X}(x) d x+\omega_{2}(y)
$$

where

$$
\begin{aligned}
& \omega_{1}(y)=\int_{y}^{\infty} \omega(y, x-y) f_{X}(x) d x, \\
& \omega_{2}(y)=\int_{y}^{\infty} \omega(y, x-y) k_{X}(x) d x .
\end{aligned}
$$

Given (10) and (11), (9) becomes

$$
m_{\delta}(u)=\int_{0}^{\infty} e^{-\delta t} f_{V}(t) \sigma_{1}(u+c t) d t+\theta \int_{0}^{\infty} e^{-\delta t} k_{V}(t) \sigma_{2}(u+c t) d t .
$$

Substituting $s=u+c t$ into (12), we have

$$
\begin{aligned}
\operatorname{cm}_{\delta}(u)= & \int_{u}^{\infty} e^{-\delta \frac{s-u}{c}} f_{V}\left(\frac{s-u}{c}\right) \sigma_{1}(s) d s \\
& +\theta \int_{u}^{\infty} e^{-\delta \frac{s-u}{c}} k_{V}\left(\frac{s-u}{c}\right) \sigma_{2}(s) d s .
\end{aligned}
$$

Theorem 1 In the risk model introduced in Section 2, the excepted discounted penalty function $m_{\delta}(u)$ satisfies the following integro-differential equation:

$$
\alpha(D) \beta(D) m_{\delta}(u)=\alpha(D)\left(\sigma_{1}(u)-\theta \sigma_{2}(u)\right)+\theta \beta(D) \gamma(D) \sigma_{2}(u),
$$

where

$$
\begin{aligned}
& \alpha(D)=\left(\frac{\lambda_{1}+\lambda_{2}+\delta}{2} I-\frac{c}{2} D\right)\left(\frac{2 \lambda_{1}+\delta}{\lambda_{1}} I-\frac{c}{\lambda_{1}} D\right)\left(\frac{2 \lambda_{2}+\delta}{\lambda_{2}} I-\frac{c}{\lambda_{2}} D\right), \\
& \beta(D)=\left(\frac{\lambda_{1}+\delta}{\lambda_{1}} I-\frac{c}{\lambda_{1}} D\right)\left(\frac{\lambda_{2}+\delta}{\lambda_{2}} I-\frac{c}{\lambda_{2}} D\right),
\end{aligned}
$$




$$
\gamma(D)=\left(2 \lambda_{1}+2 \lambda_{2}+\delta\right) I-c D
$$

$I$ and $D$ are the identity and differential operators.

Proof Case 1. $\lambda_{1} \neq \lambda_{2}$, we obtain the p.d.f. and c.d.f. of r.v. $V$,

$$
\begin{aligned}
& f_{V}(t)=\frac{\lambda_{1} \lambda_{2}}{\lambda_{2}-\lambda_{1}}\left(e^{-\lambda_{1} t}-e^{-\lambda_{2} t}\right), \\
& F_{V}(t)=1-\frac{1}{\lambda_{2}-\lambda_{1}}\left(\lambda_{2} e^{-\lambda_{1} t}-\lambda_{1} e^{-\lambda_{2} t}\right),
\end{aligned}
$$

hence

$$
\begin{aligned}
k_{V}(t) & =f_{V}(t)\left(1-2 F_{V}(t)\right) \\
& =\frac{\lambda_{1} \lambda_{2}}{\lambda_{2}-\lambda_{1}}\left(e^{-\lambda_{1} t}-e^{-\lambda_{2} t}\right)\left(\frac{2 \lambda_{1}}{\lambda_{1}-\lambda_{2}} e^{-\lambda_{2} t}-\frac{2 \lambda_{2}}{\lambda_{1}-\lambda_{2}} e^{-\lambda_{1} t}-1\right) .
\end{aligned}
$$

Let

$$
\lambda=\frac{\lambda_{1} \lambda_{2}}{\lambda_{2}-\lambda_{1}}, \quad r_{1}=\frac{2 \lambda_{1}}{\lambda_{1}-\lambda_{2}}, \quad r_{2}=\frac{2 \lambda_{2}}{\lambda_{1}-\lambda_{2}},
$$

then (13) becomes

$$
\begin{aligned}
m_{\delta}(u)= & \frac{\lambda}{c} \int_{u}^{\infty}\left(e^{-\left(\delta+\lambda_{1}\right) \frac{s-u}{c}}-e^{-\left(\delta+\lambda_{2}\right) \frac{s-u}{c}}\right)\left(\sigma_{1}(s)-\theta \sigma_{2}(s)\right) d s \\
& +\theta \frac{\lambda}{c} \int_{u}^{\infty}\left(e^{-\left(\delta+\lambda_{1}\right) \frac{s-u}{c}}-e^{-\left(\delta+\lambda_{2}\right) \frac{s-u}{c}}\right)\left(r_{1} e^{-\lambda_{2} \frac{s-u}{c}}-r_{2} e^{-\lambda_{1} \frac{s-u}{c}}\right) \sigma_{2}(s) d s \\
= & \frac{\lambda}{c} \xi(u)+\theta \frac{\lambda}{c} \eta(u),
\end{aligned}
$$

where

$$
\xi(u)=\int_{u}^{\infty}\left(e^{-\left(\delta+\lambda_{1}\right) \frac{s-u}{c}}-e^{-\left(\delta+\lambda_{2}\right) \frac{s-u}{c}}\right)\left(\sigma_{1}(s)-\theta \sigma_{2}(s)\right) d s
$$

and

$$
\begin{aligned}
\eta(u) & =\int_{u}^{\infty}\left(e^{-\left(\delta+\lambda_{1}\right) \frac{s-u}{c}}-e^{-\left(\delta+\lambda_{2}\right) \frac{s-u}{c}}\right)\left(r_{1} e^{-\lambda_{2} \frac{s-u}{c}}-r_{2} e^{-\lambda_{1} \frac{s-u}{c}}\right) \sigma_{2}(s) d s \\
& =\int_{u}^{\infty}\left(\left(r_{1}+r_{2}\right) e^{-\left(\lambda_{1}+\lambda_{2}+\delta\right) \frac{s-u}{c}}-r_{1} e^{-\left(2 \lambda_{2}+\delta\right) \frac{s-u}{c}}-r_{2} e^{-\left(2 \lambda_{1}+\delta\right) \frac{s-u}{c}}\right) \sigma_{2}(s) d s .
\end{aligned}
$$

In order to get (14), we firstly apply the operator $\beta(D)$ to both sides of (18) with respect to (w.r.t.) $u$.

Given (18), we have

$$
D \xi(u)=\int_{u}^{\infty}\left(\frac{\lambda_{1}+\delta}{c} e^{-\left(\delta+\lambda_{1}\right) \frac{s-u}{c}}-\frac{\lambda_{2}+\delta}{c} e^{-\left(\delta+\lambda_{2}\right) \frac{s-u}{c}}\right)\left(\sigma_{1}(s)-\theta \sigma_{2}(s)\right) d s
$$


and

$$
\begin{aligned}
D^{2} \xi(u)= & \int_{u}^{\infty}\left(\left(\frac{\lambda_{1}+\delta}{c}\right)^{2} e^{-\left(\delta+\lambda_{1}\right) \frac{s-u}{c}}-\left(\frac{\lambda_{2}+\delta}{c}\right)^{2} e^{-\left(\delta+\lambda_{2}\right) \frac{s-u}{c}}\right)\left(\sigma_{1}(s)-\theta \sigma_{2}(s)\right) d s \\
& -\frac{\lambda_{1}-\lambda_{2}}{c}\left(\sigma_{1}(u)-\theta \sigma_{2}(u)\right) .
\end{aligned}
$$

These allow us to derive the following result:

$$
\begin{aligned}
\beta(D) \xi(u) & =\frac{1}{\lambda_{1} \lambda_{2}}\left(\left(\lambda_{1}+\delta\right)\left(\lambda_{2}+\delta\right) I-c\left(\lambda_{1}+\lambda_{2}+2 \delta\right) D+c^{2} D^{2}\right) \\
& =\frac{c}{\lambda}\left(\sigma_{1}(u)-\theta \sigma_{2}(u)\right) .
\end{aligned}
$$

Secondly, we take the operator $\alpha(D)$ to both sides of (19) w.r.t. $u$.

From (19), we get

$$
\begin{aligned}
D \eta(u)= & \int_{u}^{\infty}\left(\left(r_{1}+r_{2}\right) \frac{\lambda_{1}+\lambda_{2}+\delta}{c} e^{-\left(\lambda_{1}+\lambda_{2}+\delta\right) \frac{s-u}{c}}-r_{1} \frac{2 \lambda_{2}+\delta}{c} e^{-\left(2 \lambda_{2}+\delta\right) \frac{s-u}{c}}\right. \\
& \left.-r_{2} \frac{2 \lambda_{1}+\delta}{c} e^{-\left(2 \lambda_{1}+\delta\right) \frac{s-u}{c}}\right) \sigma_{2}(s) d s \\
D^{2} \eta(u)= & \int_{u}^{\infty}\left(\left(r_{1}+r_{2}\right)\left(\frac{\lambda_{1}+\lambda_{2}+\delta}{c}\right)^{2} e^{-\left(\lambda_{1}+\lambda_{2}+\delta\right) \frac{s-u}{c}}-r_{1}\left(\frac{2 \lambda_{2}+\delta}{c}\right)^{2} e^{-\left(2 \lambda_{2}+\delta\right) \frac{s-u}{c}}\right. \\
& \left.-r_{2}\left(\frac{2 \lambda_{1}+\delta}{c}\right)^{2} e^{-\left(2 \lambda_{1}+\delta\right) \frac{s-u}{c}}\right) \sigma_{2}(s) d s \\
& -\left(\left(r_{1}+r_{2}\right)\left(\frac{\lambda_{1}+\lambda_{2}+\delta}{c}\right)-r_{1}\left(\frac{2 \lambda_{2}+\delta}{c}\right)-r_{2}\left(\frac{2 \lambda_{1}+\delta}{c}\right)\right) \sigma_{2}(u)
\end{aligned}
$$

and

$$
\begin{aligned}
D^{3} \eta(u)= & \int_{u}^{\infty}\left(\left(r_{1}+r_{2}\right)\left(\frac{\lambda_{1}+\lambda_{2}+\delta}{c}\right)^{3} e^{-\left(\lambda_{1}+\lambda_{2}+\delta\right) \frac{s-u}{c}}\right. \\
& \left.-r_{1}\left(\frac{2 \lambda_{2}+\delta}{c}\right)^{3} e^{-\left(2 \lambda_{2}+\delta\right) \frac{s-u}{c}}-r_{2}\left(\frac{2 \lambda_{1}+\delta}{c}\right)^{3} e^{-\left(2 \lambda_{1}+\delta\right) \frac{s-u}{c}}\right) \sigma_{2}(s) d s \\
& -\left(\left(r_{1}+r_{2}\right)\left(\frac{\lambda_{1}+\lambda_{2}+\delta}{c}\right)^{2}-r_{1}\left(\frac{2 \lambda_{2}+\delta}{c}\right)^{2}-r_{2}\left(\frac{2 \lambda_{1}+\delta}{c}\right)^{2}\right) \sigma_{2}(u) \\
& -\left(\left(r_{1}+r_{2}\right)\left(\frac{\lambda_{1}+\lambda_{2}+\delta}{c}\right)-r_{1}\left(\frac{2 \lambda_{2}+\delta}{c}\right)-r_{2}\left(\frac{2 \lambda_{1}+\delta}{c}\right)\right) D \sigma_{2}(u) .
\end{aligned}
$$

Hence

$$
\begin{aligned}
\alpha(D) \eta(u)= & \frac{1}{2 \lambda_{1} \lambda_{2}}\left\{\left(\lambda_{1}+\lambda_{2}+\delta\right)\left(2 \lambda_{1}+\delta\right)\left(2 \lambda_{2}+\delta\right) \eta(u)\right. \\
& -c\left(2\left(\lambda_{1}+\lambda_{2}+\delta\right)^{2}+\left(2 \lambda_{1}+\delta\right)\left(2 \lambda_{2}+\delta\right)\right) D \eta(u) \\
& \left.+3 c^{2}\left(\lambda_{1}+\lambda_{2}+\delta\right) D^{2} \eta(u)-c^{3} D^{3} \eta(u)\right\} \\
= & \frac{c}{\lambda}\left(\left(2 \lambda_{1}+2 \lambda_{2}+\delta\right) \sigma_{2}(u)-c D \sigma_{2}(u)\right) \\
= & \frac{c}{\lambda} \gamma(D) \sigma_{2}(u) .
\end{aligned}
$$


Applying the operator $\alpha(D) \beta(D)$ to both sides of (17), we can obtain

$$
\begin{aligned}
\alpha(D) \beta(D) m_{\delta}(u) & =\alpha(D) \beta(D)\left(\frac{\lambda}{c} \xi(u)+\theta \frac{\lambda}{c} \eta(u)\right) \\
& =\alpha(D)\left(\sigma_{1}(u)-\theta \sigma_{2}(u)\right)+\theta \beta(D) \gamma(D) \sigma_{2}(u)
\end{aligned}
$$

Case 2. $\lambda_{1}=\lambda_{2}=\lambda$, we know

$$
\begin{aligned}
& f_{V}(t)=\lambda^{2} t e^{-\lambda t} \\
& F_{V}(t)=1-(1+\lambda t) e^{-\lambda t}, \\
& k_{V}(t)=f_{V}(t)\left(1-2 F_{V}(t)\right)=2 \lambda^{2}(1+\lambda t) t e^{-2 \lambda t}-\lambda^{2} t e^{-\lambda t},
\end{aligned}
$$

then (13) becomes

$$
m_{\delta}(u)=\frac{\lambda^{2}}{c} D \xi(u)+\theta \frac{\lambda^{2}}{c} D \eta(u)
$$

where

$$
D \xi(u)=\int_{u}^{\infty} \frac{s-u}{c} e^{-(\delta+\lambda) \frac{s-u}{c}}\left(\sigma_{1}(s)-\theta \sigma_{2}(s)\right) d s
$$

and

$$
D \eta(u)=\int_{u}^{\infty}\left(\lambda\left(\frac{s-u}{c}\right)^{2}+\frac{s-u}{c}\right) e^{-(\delta+2 \lambda) \frac{s-u}{c}} \sigma_{2}(s) d s .
$$

When repeating a similar procedure to Case 1, the following formulae can be obtained:

$$
\begin{aligned}
\beta(D) D \xi(u) & =\frac{c}{\lambda^{2}}\left(\sigma_{1}(u)-\theta \sigma_{2}(u)\right), \\
\alpha(D) D \eta(u) & =\frac{c}{\lambda^{2}}\left((\delta+4 \lambda) \sigma_{2}(u)-c D \sigma_{2}(u)\right) \\
& =\frac{c}{\lambda^{2}} \gamma(D) \sigma_{2}(u) .
\end{aligned}
$$

Given (25) and (26), applying the operator $\alpha(D) \beta(D)$ to both sides of (24), we can derive

$$
\begin{aligned}
\alpha(D) \beta(D) m_{\delta}(u) & =\alpha(D) \beta(D) D\left(\frac{\lambda^{2}}{c} \xi(u)+\theta \frac{\lambda^{2}}{c} \eta(u)\right) \\
& =\alpha(D)\left(\sigma_{1}(u)-\theta \sigma_{2}(u)\right)+\theta \beta(D) \gamma(D) \sigma_{2}(u) .
\end{aligned}
$$

Hence, the integro-differential equation is true.

\section{The Laplace transform of $m_{\delta}(u)$ for $\delta=0$}

Throughout this paper we denote the Laplace transform of a function $f(x)$ by

$$
f^{\prime \prime}(s)=\int_{0}^{\infty} e^{-s x} f(x) d x
$$


Before deriving the Laplace transform of Gerber-Shiu function, one important step is to develop Lundberg's fundamental equation and examine its properties.

To derive Lundberg's fundamental equation, we consider the process

$$
\left\{\exp \left(-\delta \sum_{i=1}^{k} V_{i}+s U_{k}\right), k=0,1, \ldots\right\}, \quad \text { for } s>0
$$

It is a martingale if and only if

$$
E\left(e^{-\delta V} e^{s(c V-X)}\right)=1
$$

which corresponds to Lundberg's fundamental equation, see Gerber and Shui [9]. Due to (3), (4) and (5), (27) can be written as

$$
\begin{aligned}
E\left(e^{-\delta V} e^{s(c V-X)}\right)= & \int_{0}^{\infty} \int_{0}^{\infty} e^{t(c s-\delta)} e^{-s x} f_{X, V}(x, t) d x d t \\
& +\theta \int_{0}^{\infty} \int_{0}^{\infty} e^{t(c s-\delta)} e^{-s x} k_{X}(x) k_{V}(t) d x d t \\
= & f_{X}^{*}(s) f_{V}^{*}(\delta-c s)+\theta k_{X}^{*}(s) k_{V}^{*}(\delta-c s) \\
= & 1 .
\end{aligned}
$$

From (15), (16) and (22), (23), we conclude that $f_{V}^{*}(\delta-c s)$ and $k_{V}^{*}(\delta-c s)$ have the same form in Case 1 or Case 2,

$$
f_{V}^{*}(\delta-c s)=\left(\frac{\lambda_{1}+\delta}{\lambda_{1}}-\frac{c}{\lambda_{1}} s\right)^{-1}\left(\frac{\lambda_{2}+\delta}{\lambda_{2}}-\frac{c}{\lambda_{2}} s\right)^{-1}=\frac{1}{\beta(s)}
$$

and

$$
\begin{aligned}
k_{V}^{*}(\delta-c s) & =\frac{\left(2 \lambda_{1}+2 \lambda_{2}+\delta\right)-c s}{\alpha(s)}-\frac{1}{\beta(s)} \\
& =\frac{\gamma(s)}{\alpha(s)}-\frac{1}{\beta(s)} .
\end{aligned}
$$

Thereby, (28) is equivalent to

$$
\alpha(s) \beta(s)-\alpha(s) f_{X}^{*}(s)-\theta(\beta(s) \gamma(s)-\alpha(s)) k_{X}^{*}(s)=0 .
$$

To derive the expression of $m_{\delta}^{*}(s)$, we need to know the number of roots in the right-halfplane of Lundberg's fundamental equation (28). For $\delta=0$ and $\theta \neq 0$, by Theorem 1 of Klimenok [10], we can determine the number of roots to (28) with a positive real part. However, for $\delta>0$ and $\theta \neq 0$ we do not reach the conclusion about it.

Lemma 1 For $\delta=0$ and $\theta \neq 0$, Lundberg's fundamental equation (28) has exactly 4 roots denoted by $\left\{\rho_{i}, i=1, \ldots, 4\right\}$ with $\operatorname{Re}\left(\rho_{i}\right)>0$ and a 5 th root $\rho_{5}=0$.

Proof Let $z(s)=\frac{k-s}{k}, C_{k}=\{s:|z(s)|=1\}$. 
Firstly, $\alpha(s) \beta(s)$ and $\alpha(s) f_{X}^{*}(s)+\theta(\beta(s) \gamma(s)-\alpha(s)) k_{X}^{\prime \prime}(s)$ are analytic inside the unit contour $C_{k}$ and continuous on $C_{k}$. Let $k \rightarrow \infty$ and denote by $C$ the limiting contour. We want to show

$$
|\alpha(s) \beta(s)|>\left|\alpha(s) f_{X}^{*}(s)-\theta(\beta(s) \gamma(s)-\alpha(s)) k_{X}^{*}(s)\right|,
$$

which is equivalent to

$$
\left|f_{X}^{*}(s)\right| \frac{1}{|\beta(s)|}+\left|\theta k_{X}^{*}(s)\right| \frac{|\beta(s) \gamma(s)-\alpha(s)|}{|\alpha(s)||\beta(s)|}<1,
$$

$\frac{1}{|\beta(s)|}$ and $\frac{|\beta(s) \gamma(s)-\alpha(s)|}{|\alpha(s)||\beta(s)|}$ are the ratios of polynomials with a strictly higher degree at the denominator. From the definitions, we have $\left|f_{X}^{*}(s)\right| \leq 1$ and $\left|\theta k_{X}^{*}(s)\right| \leq 1$. Hence

$$
\left|f_{X}^{*}(s)\right| \frac{1}{|\beta(s)|}+\left|\theta k_{X}^{*}(s)\right| \frac{|\beta(s) \gamma(s)-\alpha(s)|}{|\alpha(s)||\beta(s)|} \rightarrow 0
$$

on $C$ (excluding $s=0)$. Moreover, due to (4),

$$
\begin{aligned}
& \frac{d}{d z}\left(1-\left|f_{X}^{*}(k-k z)\right| \frac{1}{|\beta(k-k z)|}\right. \\
& \left.\quad-\left|\theta k_{X}^{*}(k-k z)\right| \frac{|\beta(k-k z) \gamma(k-k z)-\alpha(k-k z)|}{|\alpha(k-k z)||\beta(k-k z)|}\right)\left.\right|_{z=1} \\
& =\left.\frac{d}{d z}\left(1-E\left(e^{-k(1-z)(X-c V)}\right)\right)\right|_{z=1}=E(c V-X)>0 .
\end{aligned}
$$

Because $\alpha(s) \beta(s)$ have 5 positive roots, by Theorem 1 of Klimenok [10], we can conclude that the number of solutions to (28) inside $C$ is equal to 4 . Finally, it is clear that the 5 th root to (28) is $\rho_{5}=0$ with $\delta=0$. Hence, the conclusion is true.

In the following sections, we only consider the case that the roots $\left\{\rho_{i}, i=1, \ldots, 5\right\}$ are distinct.

Theorem 2 In the risk model introduced in Section 2, the Laplace transform of $m_{\delta}(u)$ for $\delta=0$ denoted by $m_{0}^{*}(s)$ is

$$
m_{0}^{* \prime}(s)=\frac{\tilde{\omega}(s)-\tilde{q}(s)}{\tilde{f}(s)}
$$

where

$$
\begin{aligned}
& \tilde{\omega}(s)=\alpha(s) \omega_{1}^{*}(s)+\theta(\beta(s) \gamma(s)-\alpha(s)) \omega_{2}^{*}(s), \\
& \tilde{q}(s)=\sum_{j=1}^{5} \tilde{\omega}\left(\rho_{j}\right) \prod_{k=1, k \neq j}^{5} \frac{s-\rho_{k}}{\rho_{j}-\rho_{k}}
\end{aligned}
$$

and

$$
\tilde{f}(s)=\alpha(s) \beta(s)-\alpha(s) f_{X}^{\prime \prime}(s)-\theta(\beta(s) \gamma(s)-\alpha(s)) k_{X}^{\prime \prime}(s) .
$$


Proof First, from the properties of the Laplace transform, we have

$$
\left(f^{(k)}\right)^{*}(s)=s^{k} f^{*}(s)-\sum_{l=0}^{k-1} s^{k-l-1} D^{l} f(0), \quad \text { for } k \in \mathbb{N}^{+}
$$

and

$$
\left(\int_{0}^{x} f_{1}(y) f_{2}(x-y) d y\right)^{*}(s)=f_{1}^{*}(s) f_{2}^{*}(s)
$$

In order to get (30), we must take the Laplace transform of both sides of (14).

The Laplace transform of $\alpha(D) \beta(D) m_{0}(u)$ is

$$
\alpha(s) \beta(s) m_{0}^{*}(s)+q(s)
$$

where $q(s)$ is a polynomial of degree four or less, with coefficients in terms of $c, \lambda_{1}, \lambda_{2}$, and the value of $m_{0}(0)$ and its first 4 derivatives at $u=0$.

The Laplace transform of $\alpha(D)\left(\sigma_{1}(u)-\theta \sigma_{2}(u)\right)+\theta \beta(D) \gamma(D) \sigma_{2}(u)$ is

$$
\begin{aligned}
& \left\{\alpha(s)\left[\left(m_{0}^{*}(s) f_{X}^{*}(s)+\omega_{1}^{*}(s)\right)-\theta\left(m_{0}^{*}(s) k_{X}^{*}(s)+\omega_{2}^{*}(s)\right)\right]+q_{1}(s)\right\} \\
& \quad+\theta\left\{\beta(s) \gamma(s)\left[m_{0}^{*}(s) k_{X}^{*}(s)+\omega_{2}^{*}(s)\right]+q_{2}(s)\right\}
\end{aligned}
$$

where $q_{1}(s)$ and $q_{2}(s)$ are polynomials of degree three or less, with coefficients in terms of $c, \lambda_{1}, \lambda_{2}$.

For simplicity, we define the following functions:

$$
\begin{aligned}
& \tilde{\omega}(s)=\alpha(s) \omega_{1}^{*}(s)+\theta(\beta(s) \gamma(s)-\alpha(s)) \omega_{2}^{*}(s), \\
& \tilde{q}(s)=q(s)-q_{1}(s)-\theta q_{2}(s)
\end{aligned}
$$

and

$$
\tilde{f}(s)=\alpha(s) \beta(s)-\alpha(s) f_{X}^{\prime \prime}(s)-\theta(\beta(s) \gamma(s)-\alpha(s)) k_{X}^{* \prime}(s),
$$

then

$$
\begin{aligned}
\alpha(s) \beta(s) m_{0}^{*}(s)+q(s)= & \left(\alpha(s) f_{X}^{* \prime}(s)+\theta(\beta(s) \gamma(s)-\alpha(s)) k_{X}^{* \prime}(s)\right) m_{0}^{\prime \prime}(s) \\
& +\omega(s)+q_{1}(s)+\theta q_{2}(s) .
\end{aligned}
$$

Hence

$$
m_{0}^{*}(s)=\frac{\tilde{\omega}(s)-\tilde{q}(s)}{\tilde{f}(s)} .
$$

Because $m_{0}^{*}(s)$ is finite for $\operatorname{Re}(s) \geq 0$, the numerator on the right-hand side of (31) must be zero whenever the denominator is zero. From Lemma 1, it follows that $\tilde{q}(s)$ is the collocation polynomial of function $\tilde{\omega}(s)$ with respect to $\left\{\rho_{i}, i=1, \ldots, 5\right\}$. Then, by the Lagrange 
interpolation formula, we obtain

$$
\tilde{q}(s)=\sum_{j=1}^{5} \tilde{\omega}\left(\rho_{j}\right) \prod_{k=1, k \neq j}^{5} \frac{\rho_{k}-s}{\rho_{k}-\rho_{j}}
$$

Thereby, we complete the proof.

\section{Exponential claim size}

In this section, we assume that the individual claim size follows an exponential distribution with parameter $\mu$.

Let

$$
f_{X}(x)=\mu e^{-\mu x}
$$

and

$$
\begin{aligned}
k_{X}(x) & =f_{X}(x)\left(1-2 F_{X}(x)\right) \\
& =2 \mu e^{-2 \mu x}-\mu e^{-\mu x},
\end{aligned}
$$

then

$$
\begin{aligned}
& f_{X}^{*}(s)=\frac{\mu}{\mu+s}, \\
& k_{X}^{* \prime}(s)=\frac{2 \mu}{2 \mu+s}-\frac{\mu}{\mu+s} .
\end{aligned}
$$

In order to get the expression of the ruin probability, we consider a special case of the expected penalty function with $\omega(x, y)=1$ for all $x, y>0$.

Corollary 1 In the risk model introduced in Section 2, if $X \sim \operatorname{Exp}(\mu)$ and $\omega(x, y)=1$, the Laplace transform of ruin probability denoted by $\psi^{*}(s)$ is

$$
\psi^{*}(s)=\frac{\widehat{\omega}(s)-\widehat{q}(s)}{\widehat{f}(s)}
$$

Proof Given $\omega(x, y)=1$, (31) and (32), we know

$$
\begin{aligned}
& m_{0}^{*}(s)=\psi^{*}(s), \\
& \tilde{\omega}(s)=(2 \mu+s) \alpha(s)+\theta \mu(\beta(s) \gamma(s)-\alpha(s)),
\end{aligned}
$$

and

$$
\tilde{f}(s)=\alpha(s) \beta(s)-\alpha(s) \frac{\mu}{\mu+s}-\theta(\beta(s) \gamma(s)-\alpha(s))\left(\frac{2 \mu}{2 \mu+s}-\frac{\mu}{\mu+s}\right) .
$$

For simplicity, to invert the Laplace transform of $\psi^{*}(s)$, we multiply $(\mu+s)(2 \mu+s)$ on $\tilde{f}(s)$, $\tilde{\omega}(s), \tilde{q}(s)$ respectively, yielding

$$
\widehat{f}(s)=(\mu+s)(2 \mu+s) \tilde{f}(s),
$$




$$
\begin{aligned}
& \widehat{\omega}(s)=(\mu+s)(2 \mu+s) \tilde{\omega}(s), \\
& \widehat{q}(s)=(\mu+s)(2 \mu+s) \tilde{q}(s) .
\end{aligned}
$$

So

$$
\psi^{*}(s)=\frac{\widehat{\omega}(s)-\widehat{q}(s)}{\widehat{f}(s)} .
$$

Example 1 For the numerical results, we choose $\lambda_{1}=1, \lambda_{2}=1, \mu=1$ and the premium rate $c=1$. We can invert the Laplace transform in (35) leading to $\psi(u)$. In Table 1 , the analytic expressions of $\psi(u)$ are provided for differential dependence parameters: $\theta=-1 ;-0.5 ;-0.25 ; 0 ; 0.25 ; 0.5$ and 1 , respectively.

From analytic expressions of ruin probability in Table 1, the resulting ruin probabilities are depicted in Figure 1. We can see that for fixed value of initial surplus $u$ and the impact of the dependence parameters range from -1 to 1 , the ruin probabilities decrease.

Table 1 Analytic expressions of ruin probability

\begin{tabular}{ll}
\hline $\boldsymbol{\theta}$ & Expressions for the ruin probability $\boldsymbol{\psi ( u )}$ \\
\hline-1 & $0.5201 \exp (-0.5214 u)-0.02806 \exp (-2.2149 u)$ \\
-0.5 & $0.4665 \exp (-0.5661 u)-0.01537 \exp (-2.0653 u)$ \\
-0.25 & $0.4372 \exp (-0.5910 u)-0.008086 \exp (-2.0335 u)$ \\
0 & $0.3820 \exp (-0.6180 u)$ \\
0.25 & $0.3557 \exp (-0.6474 u)+0.008384 \exp (-1.9646 u)$ \\
0.5 & $0.3270 \exp (-0.6794 u)+0.01841 \exp (-1.9271 u)$ \\
1 & $0.2600 \exp (-0.7534 u)+0.04565 \exp (-1.8434 u)$ \\
\hline
\end{tabular}

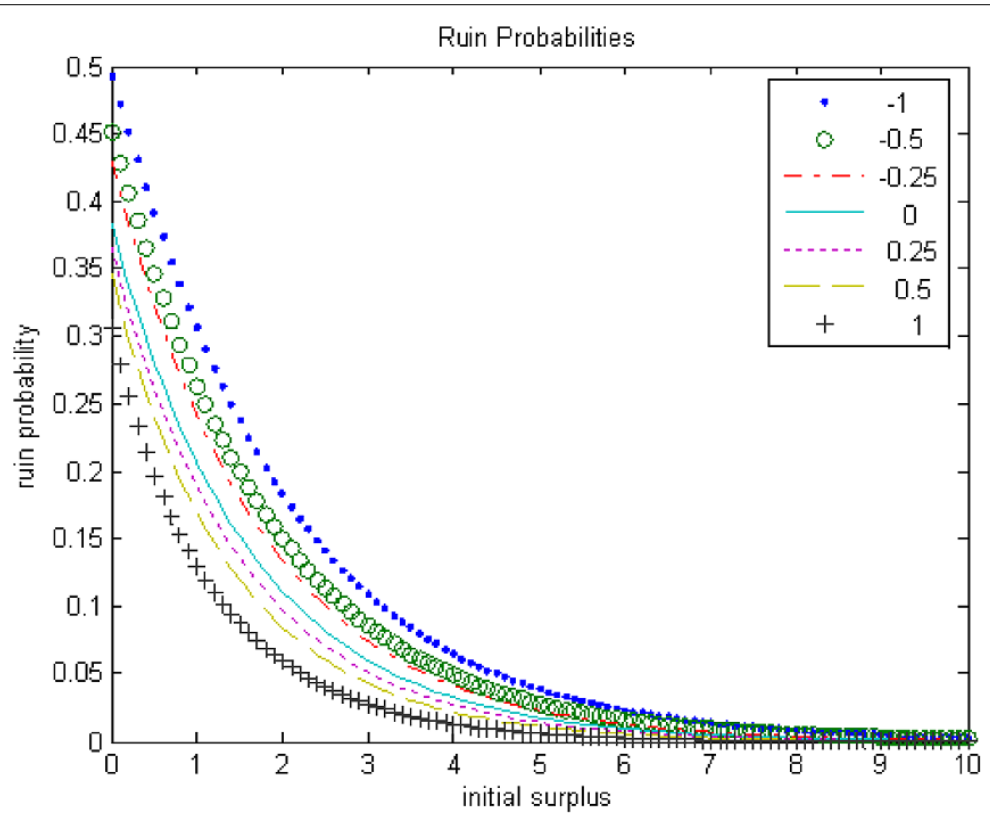

Figure 1 The result of ruin probability. 
Competing interests

The authors declare that they have no competing interests.

\section{Author's contributions}

WY and HX work together to complete the composition of this paper. HX finished the draft and WY modified it.

\section{Author details}

${ }^{1}$ School of Mathematics and Statistics, Chongqing University of Technology, Chongqing 400054, P.R. China. ${ }^{2}$ Department of Risk Management and Insurance, School of Economics, Nankai University, Tianjin 300071, P.R. China.

\section{Acknowledgements}

This research was supported by the Preliminary Doctoral Program for Mathematics of Chongqing University of

Technology. The authors would like to express their thanks to the referees for helpful suggestions.

Received: 27 December 2011 Accepted: 26 June 2012 Published: 5 July 2012

\section{References}

1. Albrecher, $\mathrm{H}$, Teugels, J: Exponential behavior in the presence of dependence in risk theory. J. Appl. Probab. 43, 265-285 (2006)

2. Boudreault, $M$, et al.: On a risk model with dependence between interclaim arrivals and claim sizes. Scand. Actuar. J. 5 301-323 (2006)

3. Cossette, $\mathrm{H}$, et al.: On the compound Poisson risk model with dependence based on a generalized Farilie-Gunmbel-Morgenstern copula. Insur. Math. Econ. 43, 444-455 (2008)

4. Barges, $\mathrm{M}$, Cossette, $\mathrm{H}$ : On the moments of the aggregate discounted claims with dependence introduced by a FGM copula. Working papers (2009)

5. Dickson, D, Hipp, C: On the time to ruin for Erlang(2) risk process. Insur. Math. Econ. 29, 333-344 (2001)

6. Li, S, Garrido, J: On ruin for the Erlang(n) risk process. Insur. Math. Econ. 34, 391-408 (2004)

7. Gerber, H, Shiu, E: The time value of ruin in a Sparre Andersen model. N. Am. Actuar. J. 2, $49-69$ (2005)

8. Nelsen, R: An Introduction to Copulas, 2nd edn. Springer, New York (2006)

9. Gerber, H, Shiu, E: On the time value of ruin. N. Am. Actuar. J. 2, 1-25 (1998)

10. Klimenok, V: On the modification of Rouche's theorem for the queuing theory problems. Queueing Syst. 38, 431-434 (2001)

doi:10.1186/1029-242X-2012-156

Cite this article as: Yong and Xiang: Differential equations for ruin probability in a special risk model with FGM copula for the claim size and the inter-claim time. Journal of Inequalities and Applications 2012 2012:156.

\section{Submit your manuscript to a SpringerOpen ${ }^{\circ}$ journal and benefit from:}

- Convenient online submission

Rigorous peer review

- Immediate publication on acceptance

- Open access: articles freely available online

- High visibility within the field

- Retaining the copyright to your article 